\title{
Pressure and Its Derivative with Respect To Piston Mach number for an Oscillating Cone
}

\author{
Aysha Shabana ${ }^{1}$, Renita Monis ${ }^{2}$, Asha Crasta ${ }^{3}$, S.A.Khan ${ }^{4}$ \\ 1,2. Research Scholar, Department of Mathematics, M.I.T.E, Moodabidri \\ 3. Associate Professor, Department of Mathematics, M.I.T.E, Moodabidri \\ 4. Professor, Mechanical Engineering Department, IIUM, Kuala Lumpur, Malaysia
}

\begin{abstract}
The aim of present study is to obtain mathematical expressions for surface pressure and its derivative with respect to Piston Mach number for an oscillating cone. The effects of Mach number and it's geometry on Pressure and its derivative with respect to inertia level and incidence angle, and the results have been obtained. The non-dimensional surface pressure distribution on the cone remain linear for lower angle of incidence and Mach number, however, for higher Mach numbers non-linearity creeps in. For higher Mach number due to viscous-inviscid interaction the flow behaves differently. Also, it is observed that there is interaction between the boundary layer and entropy layer and this interaction makes the flow on the surface of the cone very complex. At large Mach numbers the aerodynamic heating will be a serious concern. At high angle theta and low Mach number shock wave will be detached. The surface pressure derivative with Mach number on the cone exhibits linear behaviour for lower Mach number and angle theta and non-linearity for large Mach number and angle theta.
\end{abstract}

Keywords: Oscillating cone, Piston Mach number, Pressure distribution, Pressure derivative.

\section{Introduction}

The expression for the pressure ratio of a steady cone at zero incidence was derived by Ghosh[1] with the bow shock attached. For an oscillating cone, we consider the similitudinal slab, orthogonal to the mean position of the oscillating cone surface, at a distance of $x^{1}$ from the apex. The cone is non-slender. It oscillates in pitch with small amplitude and small frequency. Thus the flow can be assumed quasi-steady and quasiaxisymmetric. As the cone moves with a Mach number of $\mathrm{M} \infty$, into the slab, the Mach number of the equivalent piston is $\mathrm{M}_{\mathrm{p}}$. Ghosh's [1] large deflection similitude giving the pressure ratio expression, is valid for a quasicone provided the Mach number behind the shock $\mathrm{M}_{2} \geq 2.5$. This constraint is necessary wherever there are Mach waves in the flow. Therefore, it is needed for the validity of Ghosh's similitude in the case of an oscillating cone too.

Ghosh [1] has derived a similarity and two resulting similititudinal parameters oscillating delta wings at high angle of incidence with shock wave being attached. This similitude given by Ghosh is been extended by Crasta and Khan to hypersonic/ supersonic flows for a plane surface wedge ([2],[7],[8]]) and Non planar wedge ([9],[10],[11]) The stableness derivatives in roll and pitch of a delta wing whose leading edge is straight [5] and ([13],[16]) and leading edges curved for supersonic flows ([13],[16]), and hypersonic flows ([13],[14],[15],,[17],[4],[6]) have been obtained by Crasta and Khan. In the present paper an attempt is made to calculate pressure and its derivative with Piston Mach number and the outcomes of pressure ratio versus Mach number and incidence angle are obtained.

\section{Analysis}

The expression for the pressure ratio of a steady cone at zero incidence (Ghosh 1984), provided the bow shock is attached, is:

$\frac{\mathrm{P}_{\mathrm{bo}}}{\mathrm{P}}=1+\gamma \mathrm{M}_{\mathrm{po}}^{2}\left(1+\frac{1}{4} \in\right)$

Where the density ratio is:

$\epsilon=\frac{2+(\gamma-1) \mathbf{M}_{\mathbf{p o}}^{2}}{2+(\gamma+1) \mathbf{M}_{\mathbf{p o}}^{2}}$

And $\mathrm{M}_{\mathrm{po}}$ is the piston Mach number of the equivalent piston, operating in a conico-annular space, $\mathrm{P}_{\mathrm{bo}}$ is the pressure on the body (cone) surface at zero incidences.

$$
\mathbf{M}_{\mathbf{p o}}=\mathbf{M} \sin \boldsymbol{\theta}_{\mathbf{c}}
$$


Where $\Theta_{c}$ is the cone semi-vertex angle.

Now:

$\frac{\mathrm{dp}_{\mathrm{bo}}}{\mathrm{dM}_{\mathrm{po}}}=2 \gamma \mathrm{PM}_{\mathrm{po}}\left[1+\frac{1}{4}\left(1+\frac{1}{2} \mathrm{M}_{\mathrm{po}} \cdot \frac{\mathrm{d} \in}{\mathrm{dM}_{\mathrm{po}}}\right)\right]$

Where:

$\frac{d \in}{\mathbf{d M}_{\mathbf{p o}}}=\frac{-8 \mathbf{M}_{\mathrm{po}}}{\left[2+(\gamma+1) \mathbf{M}_{\mathbf{p o}}^{2}\right]^{2}}$

In this problem of an oscillating cone,

Let $\alpha$ be the pitch angle at any instant, and q be the rate of pitch (positive nose up). Therefore, the "local" piston Mach number at $\mathrm{A}$ is :

$\mathbf{M}_{\mathbf{p A}}=\frac{1}{\mathrm{a}}\left[\mathrm{U} \sin \left(\theta_{\mathrm{c}}-\alpha\right)-\cos \left(\varnothing-\theta_{\mathrm{c}}\right) \cdot \frac{\left(\mathrm{x}-\mathrm{x}_{\mathbf{0}}\right)}{\cos \emptyset} \cdot \mathbf{q}\right]$

Let $\mathrm{B}$ be a point in a cross-sectional plane in the same axial location $\mathrm{x}$. if $\psi$ is the azimuthal angle subtended at the centre, by an infinitesimal area element at B, then :

Since $\alpha<<1$ :

$$
M_{p B}=\frac{1}{a}\left[U \sin \left(\theta_{c}-\alpha \cos \psi\right)-\frac{\cos \left(\emptyset-\theta_{c}\right)\left(x-x_{0}\right)}{\cos \emptyset} \cos \psi \cdot q\right]
$$

$$
\begin{aligned}
& M_{p B}=\frac{1}{a_{\square}}\left[U \sin \theta_{c-} \alpha U \cos \theta_{c} \cos \psi-\left(x-x_{o}\right)\left(\cos \emptyset \cos \theta_{c}+\sin \emptyset \sin \theta_{c}\right) q \cos \psi\right] \\
& =\frac{1}{a}\left[U \sin \theta_{c-} \alpha U \cos \theta_{c} \cos \psi-\left(x-x_{o}\right)\left(1+\tan \emptyset \tan \theta_{c}\right) q \cos \psi\right]
\end{aligned}
$$

The aerodynamic pitching derivatives are defined in the limit $\alpha$ and $\mathrm{q}$ tending to zero. We note that:

$$
\left[\mathbf{M}_{\mathrm{pB}}\right]_{\alpha} \mathbf{q} \rightarrow \mathbf{o}=\mathbf{M} \sin \theta_{\mathrm{c}}
$$

For an oscillating cone the flow is not axisymmetric, and the pressure and density very near the body surface, are functions of $\mathrm{x}$ and $\psi$. The pressure and density at any point $\mathrm{B}$, can be assumed to be functions of the instantaneous "local" piston March number, $\mathrm{M}_{\mathrm{pB}}$, since the flow is considered quasi-steady and quasiaxisymmetric. The density ratio which obtains at any point B will be termed the "local" density ratio.

If $\in^{1}$ is the local density ratio, then Eq. (1) and the afore - mentioned assumption give the local pressure $P_{b}$ as:

$\frac{\mathbf{P}_{\mathbf{b}}}{\mathbf{P}}=\mathbf{1}+\gamma \mathbf{M}_{\mathbf{p B}}^{2}\left(1+\frac{1}{4} \in^{1}\right)$

Where:

$\epsilon^{1}=\frac{2+(\gamma-1) \mathbf{M}_{\mathrm{pB}}^{2}}{2+(\gamma+1) \mathbf{M}_{\mathrm{pB}}^{2}}$

$\frac{\mathbf{d} \epsilon^{1}}{\mathbf{d M}_{\mathrm{pB}}}=\frac{-\mathbf{8}^{\mathrm{M}} \mathbf{p B}}{\left[2+\left((\gamma+\mathbf{1}) \mathbf{M}_{\mathrm{pB}}^{2}\right]^{2}\right.}$

In the limit $\alpha, \mathrm{q} \rightarrow 0$, substituting from Eq.(7):

$[\in] \alpha, \mathbf{q} \rightarrow \mathbf{0}=\frac{2+(\gamma-1) M^{2} \sin ^{2} \theta_{c}}{2+(\gamma+1) M^{2} \sin ^{2} \theta_{c}}=\epsilon$

And:

$\left[\frac{d \in^{1}}{\mathrm{dM}_{\mathrm{pB}}}\right] \alpha, q \rightarrow 0=\frac{-8\left(M \sin \theta_{c}\right)}{\left[2+(\gamma+1) M^{2} \sin ^{2} \theta_{c}\right]^{2}}=\frac{d \in}{\mathrm{dM}_{\mathrm{po}}}$

Differentiating Eq. (8) with respect to $\mathrm{M}_{\mathrm{pB}}$, we get:

$\left[\frac{\mathrm{dp}_{\mathrm{b}}}{\mathrm{dM}_{\mathrm{pB}}}\right]_{\alpha, q \rightarrow 0}=2 \gamma p K\left[1+\frac{1}{4}\left(\epsilon+\frac{1}{2} K \frac{d \epsilon}{\mathrm{dM}_{\mathrm{po}}}\right)\right]$

Where $\left(\frac{\mathbf{d} \in}{\mathbf{d M}_{\mathbf{p o}}}\right)$ a function of $\mathrm{K}$ as is can be seen from Eq. (12)

In the above equation (13), the subscript B has been dropped for convenience, since B is an arbitrarily chosen point on the cross-section.

Various graphs have been plotted and the results are discussed. 


\section{Results And Discussions}

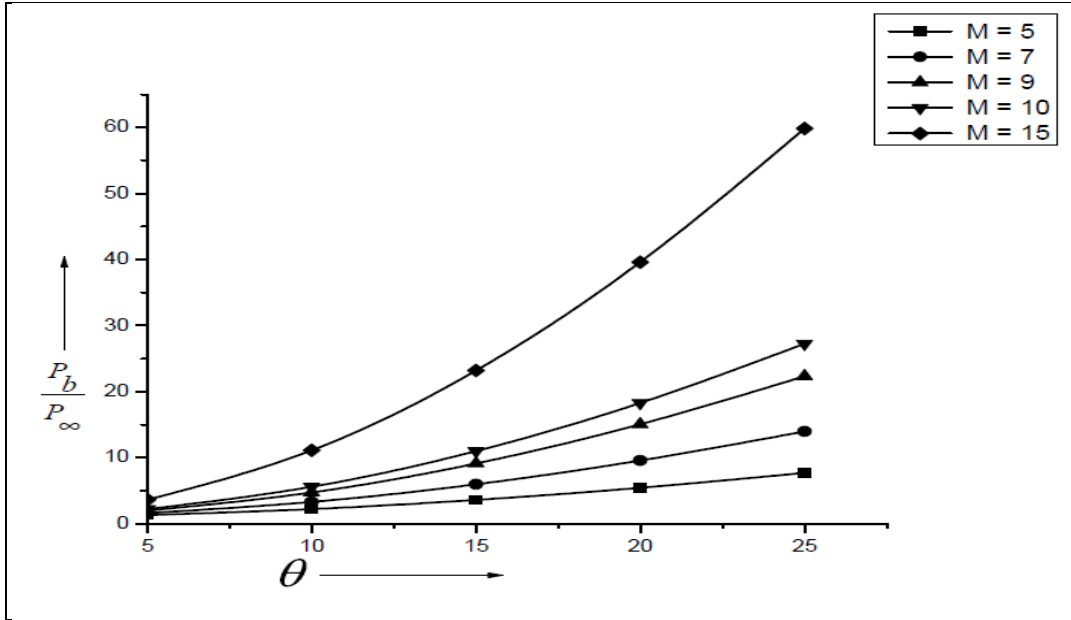

Fig.1: Surface Pressure V/S Cone angle

Fig.1 shows the pressure distribution with incidence angle for Mach Numbers in the range 5, 7, 9, 10 and 15 for an oscillating cone in hypersonic flows. Fig. 1 indicates that at lowest Mach number $\mathrm{M}=5$, the nondimensional surface pressure variation with the semi-vertex angle shows non-linearity due to its dependency with Mach number at lower inertia level. The strength of the shock wave at the cone surface, boundary layer growth and entropy layer all will affect the flow. It is also seen that for the non-linearity starts creeping in once the semi-vertex angle is 15 degree and above, due to the increase in the cone angle the flow will experience more relief and hence flow will expand more freely. This may be the reason that the flow behaviour is distinct for Mach number $\mathrm{M}=5$, as compared to the other Mach numbers.

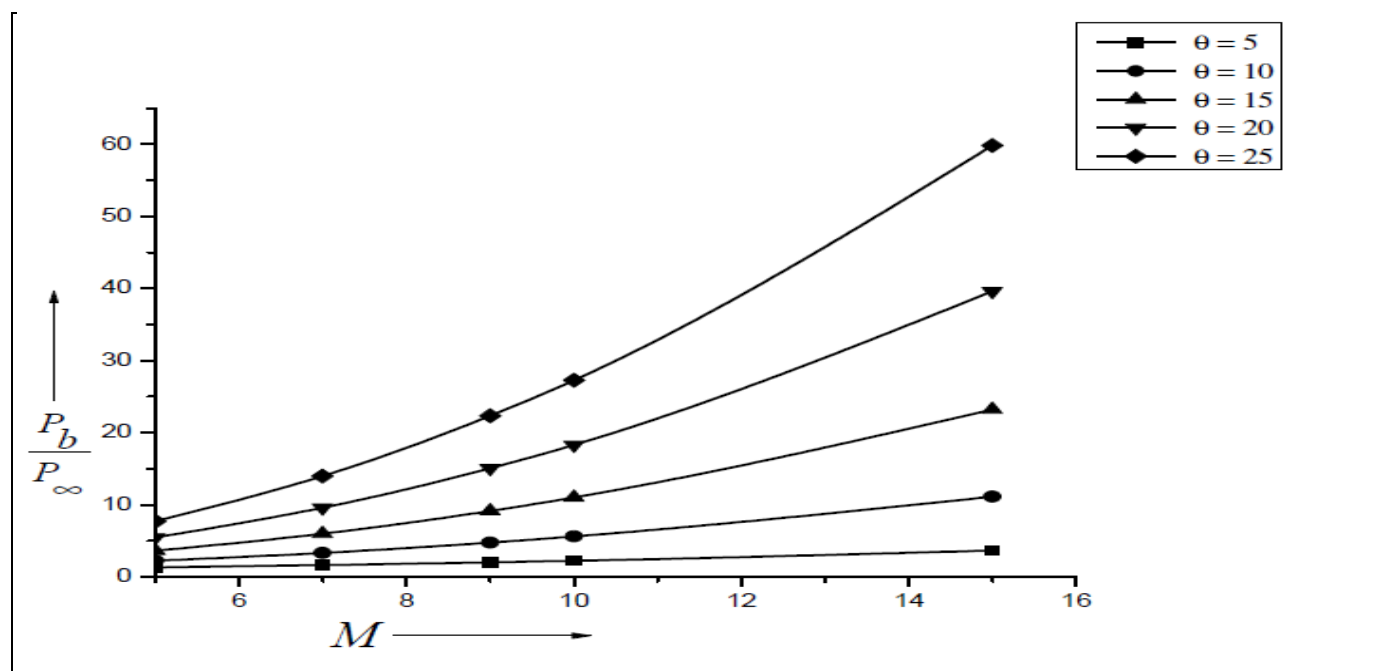

Fig.2: Pressure v/s Mach number

Fig.2 shows the variation of non-dimensional surface Pressure with Mach numbers for various angle of attacks namely 5, 10, 15, 20 and 25. It is evident from Fig.2 that for semi vertex angle of 5 degrees of the oscillating cone there is no much variation in the values of the pressure ratio. The physical reasons for this trend could be as the cone angle is small the shock wave angle will be very small, and under these circumstances the shock wave will be very closed to the surface of the cone and this will result in a single powerful shock for the entire length of the cone. When we consider the results for higher Mach numbers the trends are different when for Mach 20, and 25 as they need special treatment as at these Mach number knudson number also becomes dynamic similarity parameter and needs to be accounted, further, it is also needed to consider the effects of the low air density. Further, it is to be kept in mind that at high Mach number the air which is a non-reacting flow becomes reacting flow and the concept of continuum flow fails and to analyse the flow we need to study the flow at the molecular level that means we need to study at the microscopic level rather than at macroscopic level. 


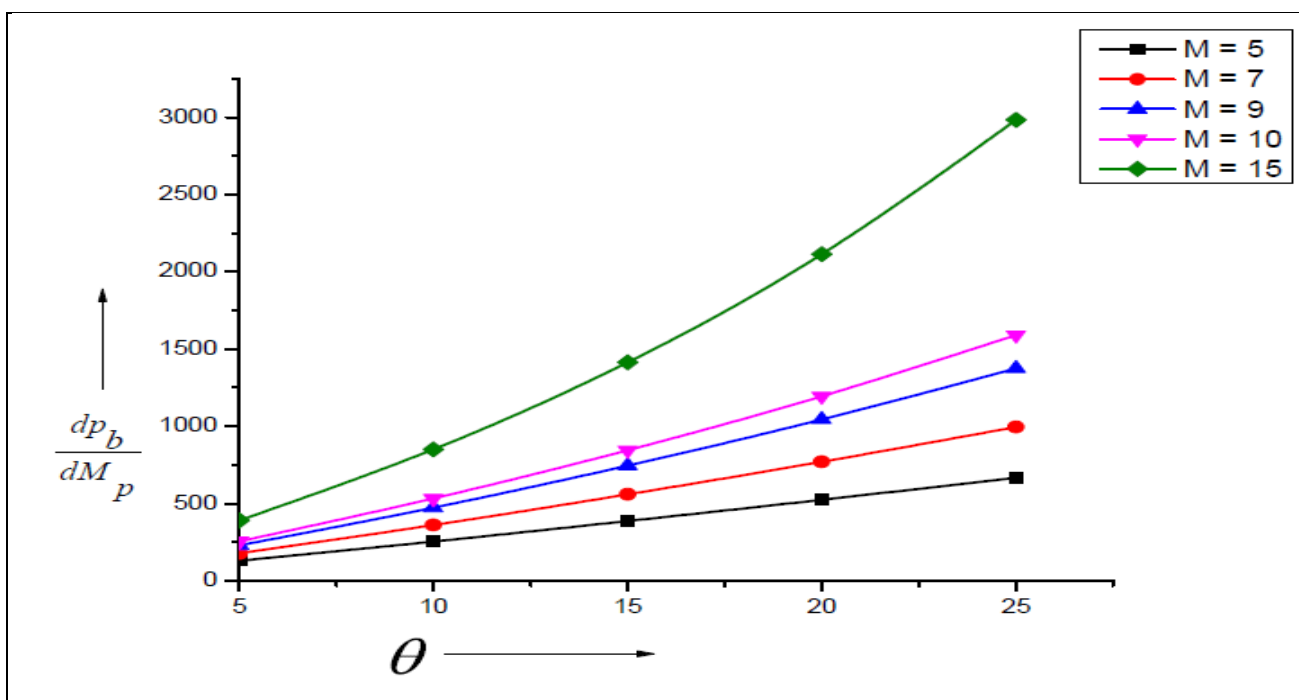

Fig.3: Variation of Surface Pressure derivative with Mach number with incidence angle

Fig.3 depicts the surface pressure derivative versus the incidence angle for Mach numbers 5,7,9,10 and 15. The linear increment of the derivative of pressure ratio for various incidence angle is evident in Fig. 3 except when the Mach number $M=15$. At semi vertex angle 5 degrees the pressure ratio derivative as its value ranging from 5-500. The surface pressure derivative for Mach number $M=15$ is distinct from all other values at lower Mach numbers in the range 5 to 10 . The reason for this trend is as was stated earlier.

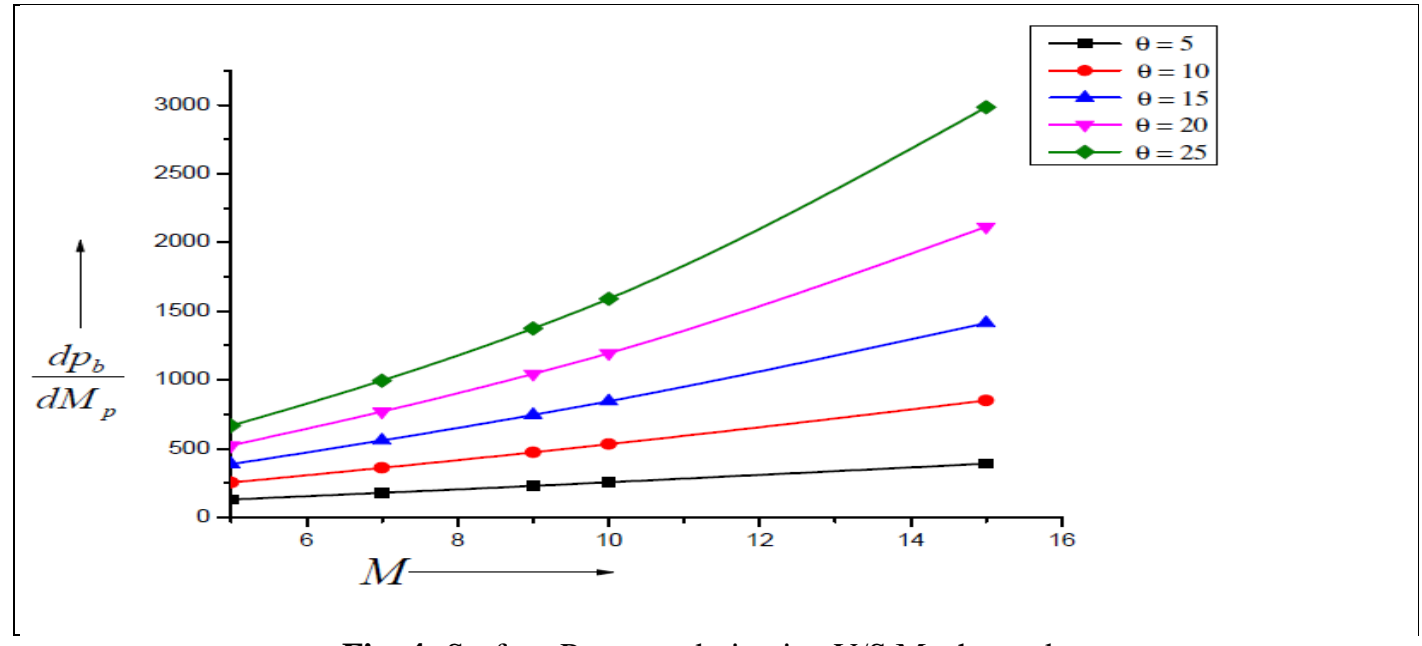

Fig. 4: Surface Pressure derivative V/S Mach number

Fig.4 represents the surface pressure derivative versus Mach number for various incidence angles $5,10,15,20$, and 25 . It is observed that the for angle 25 degrees the trend differs and lead towards non-linearity, this may due the shack wave strength, shock wave angle, boundary layer thickness, entropy layer, their interaction and viscous-inviscid interaction.

\section{Conclusion}

From the above discussions we may draw the following conclusions:

- The non-dimensional surface pressure distribution on the cone remain linear for lower angle of incidence and Mach number, however, for higher Mach numbers non-linearity creeps in.

- For higher Mach number due to viscous-inviscid interaction the flow behaves differently.

- There will be interaction between the boundary layer and entropy layer this makes flow very complicated.

- At large Mach numbers the aerodynamic heating will be a serious concern.

- At high angle theta and low Mach number shock wave will be detached.

- The surface pressure derivative with Mach number on the cone exhibits linear behaviour for lower Mach number and angle theta and non-linearity for large Mach number and angle theta. 


\section{References}

[1]. Ghosh K., (1984), Hypersonic large deflection similitude for oscillating delta wings, The Aeronautical journal, pp. 357-361.

[2]. Asha Crasta and Khan S. A., (2012), High Incidence Supersonic similitude for Planar wedge, International Journal of Engineering research and Applications, 2(5), pp. 468-471.

[3]. Asha Crasta and S. A. Khan, (2012), Estimation of Stability derivatives of an Oscillating Hypersonic delta wings with curved leading edges, IJMET, 3(3), pp. 483-492.

[4]. Asha Crasta, M. Ahmed Ali Baig, S. A. Khan, (2012), Estimation of Stability derivatives of a Delta wing in Hypersonic flow, International Journal of Emerging trends in Engineering and Developments , 6( 2), pp. 505-516.

[5]. Asha Crasta and S. A. Khan, (2012), Oscillating Supersonic delta wing with Straight Leading Edges, International Journal of Computational Engineering Research, 2(5), pp. 1226-1233.

[6]. Asha Crasta and S. A. Khan, (2014), Effect of Angle of Incidence on Stability derivatives of a wing, International Journal for Research in Applied Science and Engineering Technology, 2(V), pp. 411-422.

[7]. Asha Crasta and S. A. Khan, (2014), Hypersonic Similitude for Planar Wedges, International Journal of Advanced Research in Engineering and Technology, 5(2), pp. 16-31.

[8]. Asha Crasta and S. A. Khan, (2014), Estimation of Stability Derivatives for a Planar Wedge in the Newtonian Limit, IOSR Journal of Mathematics, 10(2), pp. 01-06.

[9]. Asha Crasta and S. A. Khan, (2014), Effect of Mach number on Stiffness and Damping Derivatives for Oscillating Hypersonic Non-Planar Wedge,IOSR Journal of Mechanical and Civil Engineering, 11(2), pp. 04-11.

[10]. Asha Crasta and S.A. Khan, (2014), Effect of Angle of Incidence on Stiffness and Damping derivatives for Oscillating Hypersonic Non-planar Wedge, International Journal for Research in Applied Science and Engineering Technology, 2(IV),pp. 229-242.

[11]. Asha Crasta and S. A. Khan,(2014), Supersonic Similitude for Oscillating Non-Planar wedge, IOSR Journal of Mathematics, 10(2), pp. 15-24.

[12]. Khan S. A. and Asha Crasta, (2010), Oscillating Supersonic delta wings with curved leading edges, Advanced Studies in Contemporary Mathematics, 20(3), pp. 359-372.

[13]. Asha Crasta and S. A. Khan (2015), "Effect of Angle of attack on Stiffness derivative of an oscillating supersonic delta wing with curved leading edges" IOSR-JMCE issue1, Volume12, December,pp12-25.

[14]. Asha Crasta and S. A. Khan, "Effect of Angle of attack on Damping derivative of a delta wing with full sine curved leading edges" IJETED Journal issue5, Volume1, December- January 2015.

[15]. Asha Crasta and S. A. Khan,(2015) "Estimation of Damping derivative of a delta wing with half sine curved leading edges" IOSR Journal of Mechanical and civil engineering, Vol.12, issue1,February ,pp40-44.

[16]. Asha Crasta and S. A. Khan (2015), "Estimation of Damping derivative in pitch of a Supersonic delta wing with curved leading edges" IOSR Journal of Journal of Mathematics , Vol. 11, issue 1, Jan-feb ,pp.07-15.

[17]. Asha Crasta and S.A.Khan (2015),"Effect of Aspect ratio with angle of attack of an oscillating Hypersonic delta wing with Straight Leading edges", Mathematical Sciences International research Journal",volume 4,issue2, pp.2833., ISSN2278-8697. 